\title{
Dielectric and plasmonic waveguides based on quantum dots embedded in polymers
}

\section{Guías de onda dieléctricas y plasmónicas basadas en puntos cuánticos embebidos en polímeros}

\author{
Isaac Suárez ${ }^{(*)}$, Henry Gordillo, Rafael Abargues, Pedro Javier Rodríguez-Cantó, \\ Sandra Albert, Juan Martínez-Pastor \\ UMDO (Unidad Asociada al CSIC-IMM), Instituto de Ciencia de los Materiales, Universidad de Valencia, PO Box 22085, \\ 46071 Valencia, Spain. \\ ${ }^{(*)}$ Email: isaac.suarez@uv.es \\ Received / Recibido: 20/09/2013. Revised / Revisado: 17/10/2013. Accepted / Aceptado: 18/10/2013. \\ DOI: http://dx.doi.org/10.7149/0PA.46.4.303
}

\begin{abstract}
:
In this work a review about the dispersion of colloidal quantum dots in polymers as method to implement active waveguides is presented. For this purpose several kinds of nanostructures (CdS, $\mathrm{CdTe}, \mathrm{CdSe}$ and $\mathrm{PbS}$ ) were embedded in PMMA to fabricate active planar waveguides in the visible and the infrared. The optimal conditions for waveguiding were obtained and the gain and attenuation carefully studied. When more than one kind of nanoparticles is dispersed in the film the structure can propagate more than one color, being it possible to obtain white light with the right proportions of red, green and blue. The losses can be improved if a polymer cladding is deposited on the QD-PMMA film, and if a pattern is defined on the film the photoluminescence of the QDs can be coupled to a 2D structure. QDs were also dispersed in SU-8 to implement 2D active waveguides. Finally, the nanocomposites are deposited on gold films with the intention to couple light and provide gain in the surface plasmon polariton.
\end{abstract}

Key words: PMMA, SU-8, Colloidal Quantum Dots, SPP, Waveguides.

\section{RESUMEN:}

En este trabajo se propone una revisión de la implementación de guías de onda activas basadas en la dispersión de puntos cuánticos coloidales (QDs) en polímeros. Para ello, guías de onda planas fueron fabricadas embebiendo distintos tipos de nanoestructuras semiconductoras con emisión en el visible e infrarrojo ( $\mathrm{CdS}, \mathrm{CdTe}, \mathrm{CdSe}, \mathrm{PbS}$ ) en PMMA, encontrándose las condiciones óptimas para el guiado de su fotoluminiscencia y sus propiedades de ganancia y atenuación. Si varios tipos de puntos son dispersados en la misma capa se propagan varios colores, siendo posible obtener luz blanca con las proporciones adecuadas de rojo, verde y azul. Asimismo, el guiado puede ser mejorado si una cubierta es depositada sobre la capa QD-PMMA, siendo posible transferir la fotoluminiscencia a una estructura 2D si se hace litografía sobre dicha capa. De la misma manera, las nanopartículas fueron dispersadas en SU-8 para realizar guías de onda activas bidimensionales. Finalmente los nanocomposites QD-PMMA fueron depositados encima de capas de oro con el fin de estudiar el polaritón de plasmón superficial propagado.

Palabras clave: PMMA, SU-8, Puntos Cuánticos Coloidales, SPP, Guías de Onda.

\section{REFERENCES AND LINKS / REFERENCIAS Y ENLACES}

[1]. G. Lifante, Integrated Photonics. Fundamentals, John Wiley \& Sons (2003). DOI

[2]. M. A. Uddin, H. P. Chan, "Materials and process optimization in the reliable fabrication of polymer photonic devices", J. Optoelectron. Adv. M. 10, 1-17 (2008). 
[3]. V. I. Klimov, "Nanocrystal quantum dots: From fundamental photophysics to multicolor lasing", Los Alamos Science 28, 214-220 (2003).

[4]. S. Maier, Plasmonics. Fundamentals and Applications, Springer (2007).

[5]. I. Suárez, H. Gordillo, R. Abargues, S. Albert, J. Martínez-Pastor, "Photoluminescence waveguiding in CdSe and CdTe QDs-PMMA nanocomposite films", Nanotechnology 22, 435202 (2011). DOI

[6]. H. Gordillo, I. Suárez, R. Abargues, P. J. Rodríguez-Cantó, S. Albert, J. P. Martínez-Pastor, "Polymer/QDs nanocomposites for wave-guiding applications", J. Nanomater. 4, 960201 (2012).

[7]. H. Gordillo, I. Suárez, R. Abargues, P. J. Rodríguez-Cantó, J. P. Martínez-Pastor, "Color tuning and white light by dispersing CdSe, CdTe and CdS in PMMA nanocomposite waveguides", IEEE Photonics J. 5, 2201412 (2013). DOI

[8]. H. Gordillo, I. Suárez, R. Abargues, P. J. Rodríguez-Cantó, G. Almuneau, J. P. Martínez-Pastor, "Quantum-dot double layer polymer waveguides by evanescent light coupling", J. Lightwave Technol. 31, 2515-2525 (2013). DOI

\section{Introducción}

La Óptica integrada está en continua investigación de nuevos materiales capaces de proveer nuevas y más eficientes funciones ópticas [1]. Para ello, la utilización de "nanocomposites" es una aproximación muy interesante. Dicho material sintético consiste en la dispersión de nanopartículas (semiconductoras, metálicas, magnéticas...) dentro de una matriz, y por tanto, combina las propiedades de las nanoestructuras con las de la propia matriz. Desde este punto de vista, la utilización de polímeros como matriz es una alternativa muy interesante para la implementación de nuevos dispositivos fotónicos [2]. Esto es debido a las facilidades tecnológicas que ofrecen los polímeros, como por ejemplo el ser depositados en capas sobre una gran variedad de substratos o el presentar propiedades litográficas. Asimismo, este tipo de materiales es especialmente útil para la implementación de guías de onda, ya que poseen unas bajas pérdidas de propagación en un gran rango de longitudes de onda (usualmente entre $400 \mathrm{~nm}$ hasta el infrarrojo), y un índice de refracción relativamente alto $(n \sim 1.5)$. De esta manera es posible realizar materiales activos basados en polímeros dispersando en ellos distintitos tipos de nanopartículas. En concreto la utilización de puntos cuánticos coloidales ("colloidal quantum dots" QD) es una alternativa muy interesante [3]. Este tipo de nanoestructuras semiconductoras combinan las propiedades de los QDs (confinamiento tridimensional de la función de onda) junto con emisión a temperatura ambiente, y la posibilidad de sintonizar su longitud de onda de emisión (o absorción) controlando el radio de la nanopartícula o su composición por métodos químicos.

En este trabajo se presenta una revisión de nuestros avances realizados en la utilización de nanocomposites QD-polímero como núcleos activos de guías de onda. Para ello distintos tipos de QDs (CdS, CdTe, CdSe y PbS) fueron dispersados en PMMA, encontrándose las condiciones óptimas de fabricación, y estudiándose sus propiedades de transmisión acoplando la luz por el canto, y su ganancia y atenuación por el método de la rendija de longitud variable. Asimismo, diseños más avanzados que incluyen cubiertas de PMMA o litografía de SU-8 fueron realizados para mejorar las prestaciones de las guías de onda. De la misma manera, los QDs fueron dispersados en SU-8 para, aprovechando sus propiedades litográficas, realizar guías de onda activas bidimensionales. Actualmente, se está continuando el trabajo depositando los nanocomposites de QD-PMMA sobre capas de oro con el fin de proveer ganancia al polaritón de plasmón superficial ("surface plasmon polariton, $\left.S P P^{\prime \prime}\right)$ que se propaga en la interfaz dieléctico metal [4].

\section{Fabricación de guías de onda}

\subsection{Puntos cuánticos coloidales}

Los QD coloidales utilizados en este trabajo consistieron en CdS, CdSe, CdTe y PbS y fueron sintetizados por vía química por el procedimiento desarrollado en [3]. Como cada 


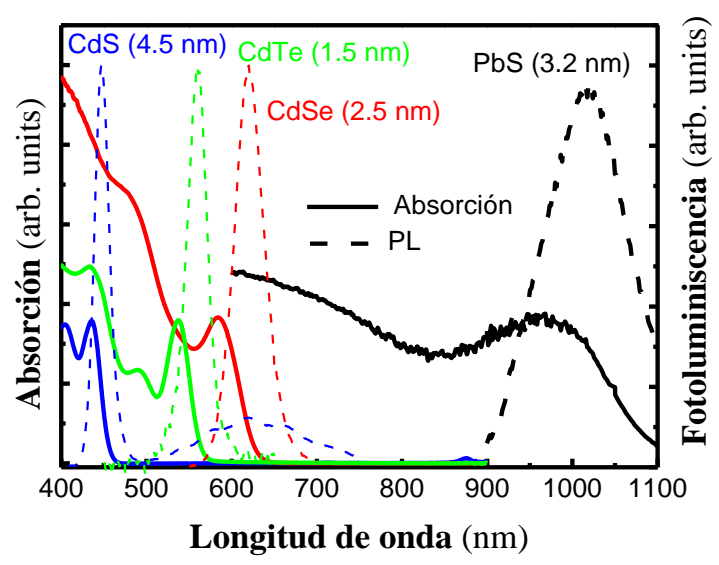

Fig. 1. Absorción (línea continua) y PL (línea discontinua) de los QDs utilizados.

QD tiene diferente composición y tamaño dan lugar a transiciones ópticas diferentes. En la Fig. 1 se muestran los espectros de absorción y fotoluminiscencia ("photoluminiscence", PL) de los QDs utilizados. Los espectros de absorción muestran el pico del excitón centrado a las longitudes de onda de $440 \mathrm{~nm}$ (CdS), $540 \mathrm{~nm}$ (CdTe), $610 \mathrm{~nm}$ (CdSe) y $1100 \mathrm{~nm}$ (PbS). A medida que la longitud de onda $(\lambda)$ está por debajo de dicho pico la absorción aumenta y aparecen estados excitados, mientras que por debajo disminuye. La emisión consiste en una banda Gaussiana separada ligeramente hacia el rojo respecto a la absorción debido al desplazamiento de Stokes [5].

\subsection{Fabricación del nanocomposite}

Para fabricar el nanocomposite las disoluciones de QDs y de polímero se mezclan con la proporción adecuada para tener la concentración deseada de QDs en la capa final.

En la Fig. 2 se puede ver la estructura de las guías de onda utilizadas en este trabajo. Para ello se han usado dos tipos de polímeros: PMMA y SU-8. En el primer caso (Fig. 2(a)) las capas se fabricaron depositando el nanocomposite (QDPMMA) sobre el substrato por centrifugado ("spin-coating") y posteriormente calentando las muestras a $80^{\circ} \mathrm{C}$ y $150^{\circ} \mathrm{C}$ durante 2 minutos. El espesor final quedaba controlado entre 1 y $3 \mu \mathrm{m}$. Los nanocomposites de SU-8 (Fig. 2(b)) siguen otro proceso para realizar la litografía. En primer lugar se depositan sobre el substrato por

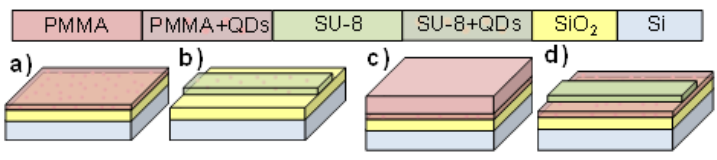

Fig. 2. Estructura de las guías de ondas.

el mismo procedimiento pero con unos calentamientos de $65^{\circ} \mathrm{C}$ y $95^{\circ} \mathrm{C}$. Después se realiza la litografía iluminando la muestra con una lámpara UV en zonas definidas por una máscara que consistió en líneas rectas de 1, 2, 4, 6, 8, 10 y $20 \mu \mathrm{m}$ separadas $50 \mu \mathrm{m}$. Posteriormente se calientan otra vez las muestras a las mismas temperaturas $\left(65^{\circ} \mathrm{C}\right.$ y $95^{\circ} \mathrm{C}$ ) y se procede a realizar el revelado. Al terminar las líneas rectas de QD-SU-8 quedaron sobre el substrato con un espesor de entre $1.5 \mathrm{y}$ $2.5 \mu \mathrm{m}$

\section{Guías de onda dieléctricas}

Para fabricar guías de onda dieléctricas los substratos utilizados sobre los cuales se depositaron los nanocomposites consistieron en obleas comerciales de $\mathrm{SiO}_{2} / \mathrm{Si}$ suministradas por CEMAT S.A. El $\mathrm{SiO}_{2}$ estaba crecido térmicamente y tenía un espesor de $2 \mu \mathrm{m}$. Debido a que el índice de refracción de los polímeros es mayor que el del $\mathrm{SiO}_{2}$ los nanocomposites pueden actuar como núcleos de las guías de onda. Asimismo el espesor de $\mathrm{SiO}_{2}$ es suficientemente grande para aislar la capa activa del $\mathrm{Si}$.

\subsection{Guías de onda de QD-PMMA}

En [5] se demostró por primera vez la posibilidad de realizar guías de onda activas mezclando QDs de CdSe en PMMA, y en [6] se extrapolaron estos resultados a otros puntos cuánticos. La capa final está formada por una matriz (polímero) junto las nanoestructuras (QDs) embebidas dentro de ella con un espesor cercano a $1 \mu \mathrm{m}$ en guías de onda operando en el visible y $3 \mu \mathrm{m}$ en el infrarrojo. El índice de refracción efectivo de la capa final varia, tanto en su parte real (confinamiento de la luz) como en su parte imaginaria (pérdidas), respecto al del PMMA inicial, siendo la desviación mayor cuanto más nanopartículas haya [5]. De esta manera, aunque el PMMA sea transparente en un amplio 


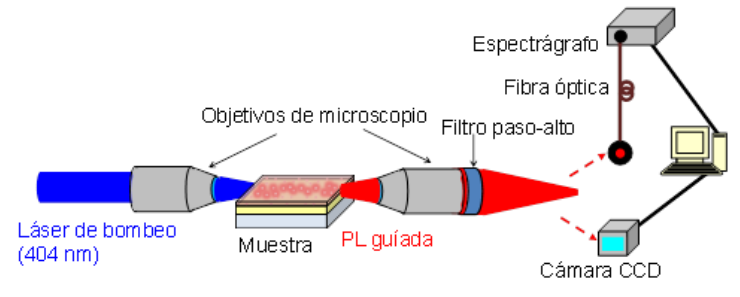

Fig. 3. Sistema experimental de acoplo de luz por el canto de la guía de ondas.

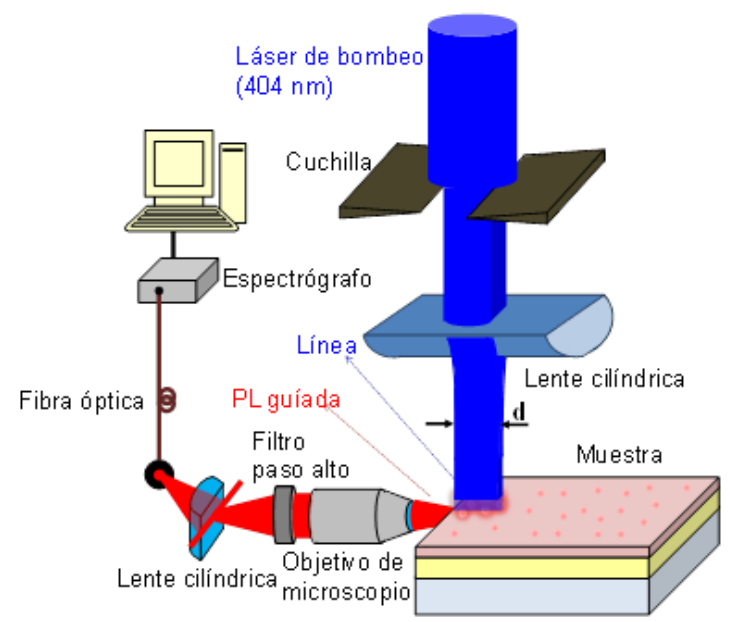

Fig. 4. Sistema experimental de acoplo por la superficie por el método de cuchilla de longitud variable.

rango de longitudes de onda, el nanocomposite presentará una absorción similar a la de los puntos cuánticos con una atenuación dependiente de la concentración de nanopartículas. Por tanto es muy importante tener en cuenta el factor de llenado de nanoestructuras dentro del polímero y sus propiedades ópticas de absorción y emisión.

Para estudiar las guías de onda se puede utilizar el sistema experimental mostrado en la Fig. 3. Consiste en acoplar la luz de un láser de bombeo (GaN, $404 \mathrm{~nm}$ ) a la guía de ondas con ayuda de un objetivo de microscopio, y recoger la luz guiada con un segundo objetivo que la enfoca en una cámara CCD o en un espectrógrafo (Stellar Net EPP2000 en el visible y Ocean Optics NirQuest512 en el IR). De esta manera, al ser la longitud de onda suficientemente baja, el láser excitará los QDs, y éstos emitirán la PL, la cual se acoplará y guiará por el nanocomposite. En este punto es importante tener en cuenta que debido precisamente a la absorción de los QDs a la longitud de onda $(\lambda)$ de bombeo, su concentración limitará longitud total de la muestra, y tal y como se encontró en [5] para unas longitudes de guías de onda prácticas (2-5 $\mathrm{mm}$ ) el factor de llenado máximo de QDs es del orden de $10^{-4}$. Sin embargo, esta limitación puede soslayarse utilizando el sistema experimental mostrado en la Fig. 4. En este caso se enfoca la luz proveniente del láser sobre la superficie de la muestra en forma de una línea con ayuda de una lente cilíndrica. De esta manera se provee una iluminación uniforme a lo largo de la superficie de la guía evitándose los problemas de absorción del láser de bombeo, y por tanto pudiéndose incrementar el factor de llenado hasta $10^{-3}$. Asimismo, con este sistema experimental se puede analizar la ganancia y las pérdidas dentro de la guía controlando la longitud $(d)$ o posición de la línea de bombeo sobre la superficie de la muestra. La ganancia se puede estudiar midiendo la PL en función de la longitud de la línea. De la misma manera las pérdidas se pueden estudiar caracterizando la PL en función de la distancia entre la línea y el borde de la muestra (manteniendo $d$ constante). Esta dependencia da lugar a una exponencial decreciente proporcional a las pérdidas de propagación. En [7] se analizó la ganancia y pérdidas de guías de onda de CdSe, CdTe y CdS en PMMA encontrándose que hay un óptimo de ganancia para $d \approx 200 \mu \mathrm{m}$ y que para longitudes de línea mayores la PL satura. Asimismo se observó que la ganancia aumenta con la concentración de puntos cuánticos. Sin embargo, las pérdidas también se incrementan de la misma manera debido a efectos de reabsorción el haber solapamiento entre los espectros de absorción y emisión (ver Fig. 1), limitando su aplicación como medios activos. Asimismo en ese trabajo se estudió el efecto de embeber varias familias (CdS, CdTe y CdSe) dentro del mismo polímero. El resultado es que la guía resultante es capaz de propagar varios colores siendo posible controlar el color final con una cantidad adecuada de cada tipo de nanopartícula (teniendo en cuenta la reabsorción entre ellas y la transferencia entre varias familias). En concreto, se demostró que con una cantidad adecuada de cada familia es posible obtener el color blanco a la salida de la guía de ondas, tal y como se puede ver en la Fig. 5, donde se muestra 


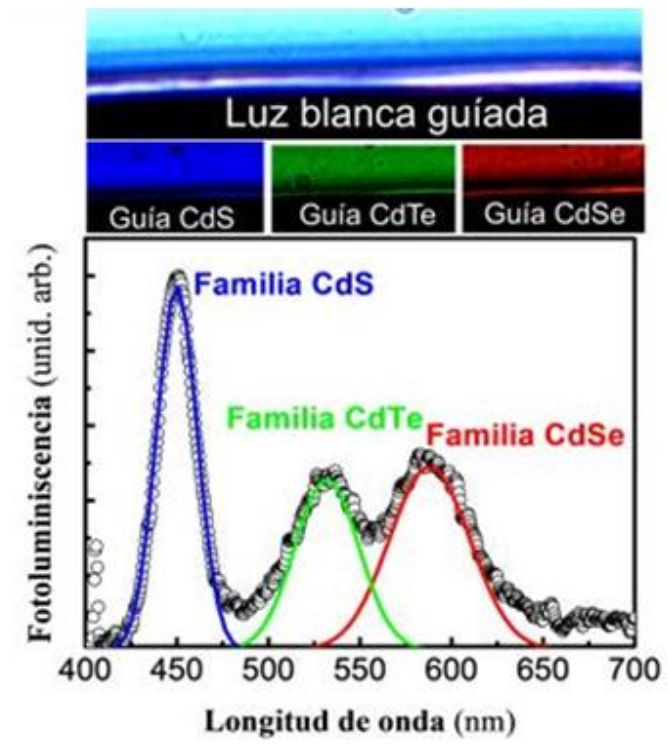

Fig. 5. Espectro de una de PMMA con puntos de CdS (azul), CdTe (verde) y rojo (CdSe) Las fotografías muestran el guiado de cada familia, así como la suma de todas que da lugar a un color blanco [6].

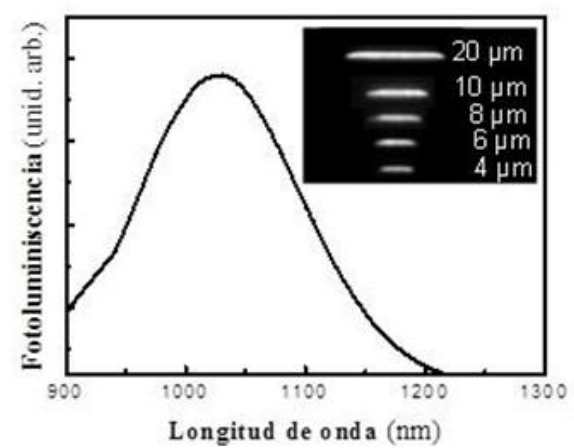

Fig. 6. Espectro de PL guiada en una guía de ondas 2D de PbS-SU-8. Las fotografías muestran la distribución modal con diferentes anchuras.

el espectro resultante y una fotografía de la PL emitida por cada familia así como la suma total.

\subsection{Guías de onda de QD-SU-8}

El PMMA es un polímero con las ventajas de un fácil procesado y una buena dispersión de los QDs dentro de ella. Sin embargo a la hora de realizar estructuras bidimiensionales presenta el inconveniente de no ser litografiable mediante litografía UV convencional. Para estos propósitos, el SU-8 es una buena alternativa. Este polímero tiene un alto índice de refracción $(n \sim 1.5)$ así como transparencia desde $500 \mathrm{~nm}$ hasta el IR, por lo que se ha utilizado en

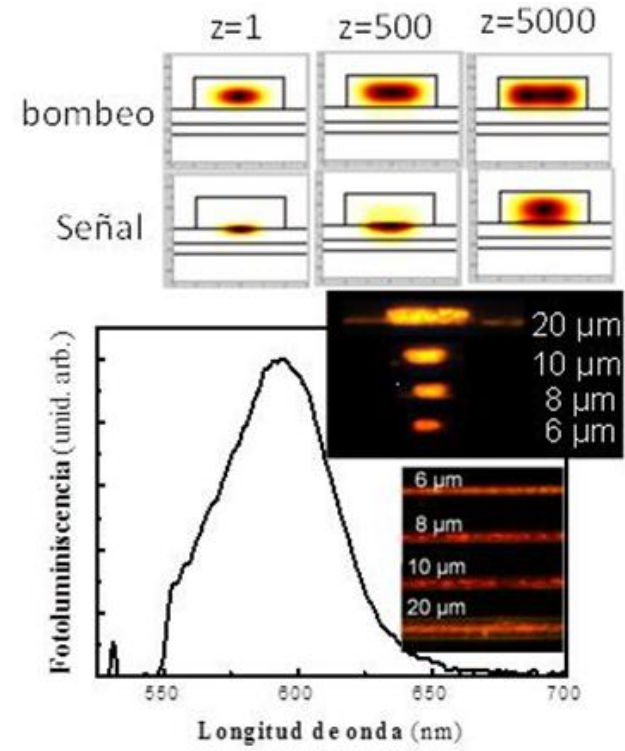

Fig. 7. Espectro de una guía bicapa SU8/CdSe-PMMA. Las fotografías muestran los modos guíados y la PL a lo largo del SU8. Las simulaciones en la parte superior exponen la evolución del láser de bombeo y señal a lo largo de la guía [8].

numerosos trabajos para implementar guías de onda. Además, en [6] se demostró por primera vez su utilización para actuar además como matriz de QDs de CdSe utilizando las estructuras mostradas en la Fig. 2(b). Para ello, fue necesario realizar el cambio de ligando correspondiente en los QDs para disolverlos en $\gamma$-butirolactona [6]. En la Fig. 6 puede ver un resultado de este tipo de guías de onda en las que se mezcló QDs de $\mathrm{PbS}$ en SU-8. El espectro corresponde a la PL guía de ondas excitada acoplando un láser de bombeo de $533 \mathrm{~nm}$ por el canto de la misma. En la fotografía las distribuciones modales a través de guías de onda de diferentes anchuras con una altura de $2 \mu \mathrm{m}$.

\subsection{Estructuras mixtas}

En [8] se analizó una estructura híbrida activa/pasiva cubriendo las capas de nanocomposite de CdSe-PMMA con una cubierta de PMMA (Fig. 2(c)) o una litografía de SU-8 (Fig. 2(d)). En el primer caso se dedujo que las características de guiado pueden mejorar, debido a que permite una mejor propagación del láser de bombeo y una recuperación de la PL guiada a través de la cubierta. En el segundo, se demostró que la PL del nanocomposite se acopla a los motivos de SU-8 realizados encima. En la 
Fig. 7 se puede ver el espectro de PL guiada excitado acoplando luz por el canto de la guía de ondas, junto con fotografías de su distribución modal y de la luz propagada por las estructuras visto desde arriba. Los espesores de nanocomposite y SU-8 fueron de $600 \mathrm{~nm}$ y 2.5 $\mu \mathrm{m}$ respectivamente. Este tipo de estructuras fueron rigurosamente simuladas utilizando un modelo que estudiaba el acoplo de unas capas a otras por el método de propagación del haz. En las imágenes superiores se puede ver la evolución de los haces de bombeo y señal para distintas distancias a lo largo de la guía (z). El haz de bombeo se acopla al modo fundamental y a medida que se propaga va excitando los QDs en el PMMA, acoplándose éstos a los motivos de SU8.

\section{Guías de onda plasmónicas}

Un polaritón de plasmón superficial (o "surface plasmon polariton, SPP") consiste en la propagación de un haz de luz entre un metal y un dieléctrico a través de las oscilaciones de electrones en el metal [7]. Debido a esta naturaleza híbrida este tipo de ondas tiene propiedades muy interesantes como el confinamiento por debajo del límite de difracción o una alta sensibilidad al medio que le rodea. Sin embargo, al ser el metal un medio con altas pérdidas óhmicas presenta una alta atenuación y la luz tiene una longitud de propagación muy reducida. Para soslayar este problema una opción es utilizar un dieléctrico adyacente al metal con ganancia, de manera que así compense las pérdidas en el metal. Con ello, en este trabajo y dentro del proyecto Europeo Navolchi (288869) se propone utilizar nanocomposites de QD-PMMA (o QD-SU8) para proveer ganancia en un SPP que se propaga en capas de oro. El diseño es análogo al mostrado en la Fig. 2(a) con una capa de oro evaporada encima del $\mathrm{SiO}_{2}$ o entre dos capas de PMMA. Como resultados preliminares en la Fig. 8 se muestra las simulaciones realizadas por el método de transferencia de matrices [1] de los modos TE y TM propagados en una estructura Aire/PMMA $/ \mathrm{Au} / \mathrm{SiO}_{2} / \mathrm{Si}$ a $\lambda=600$ nm. Los espesores de las capas fueron $1 \mu \mathrm{m}$ en el PMMA y $30 \mathrm{~nm}$ en el Au. El modo TE es fotónico y está

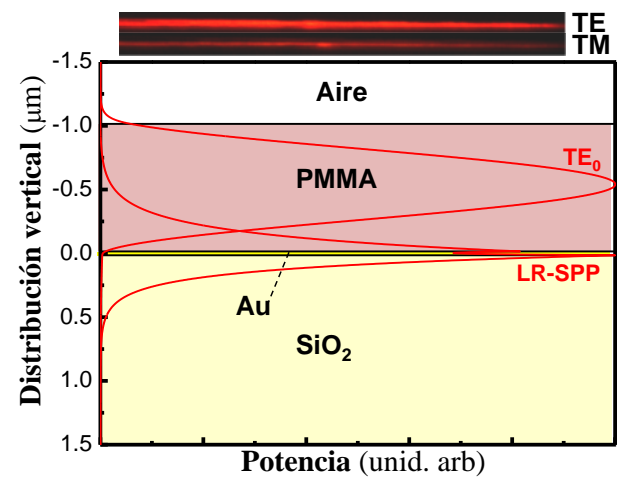

Fig. 8. Simulación de los modos propagados en una guías de ondas plasmónica. Los paneles superiores muestran fotografías del guiado en las dos polarizaciones.

centrado en el dieléctrico (PMMA), mientras que el modo TM es plasmónico y está centrado en el metal $(\mathrm{Au})$. En la parte superior se pueden ver fotografías de los modos caracterizando las muestras con el sistema experimental de la Fig. 4. Se puede observar tienen diferente sección transversal.

\section{Conclusiones}

Los nanocomposites de puntos cuánticos embebidos en polímero son unos materiales muy interesantes, no solo para las guías de onda dieléctricas y plasmónicas, sino para la Óptica Integrada en general. En este trabajo se muestran nuestros avances en guías planas y bidimensionales que pueden guiar la PL desde el visible al infrarrojo. La limitación reside en el solapamiento entre las bandas de absorción y emisión que restringen su aplicación como medios amplificadores. Pero actualmente se están utilizado otro tipo de nanostructuras que consisten en hilos cuánticos coloidales núcleo corteza ("core shell quanutm rods"), las cuales no tienen ese inconveniente $\mathrm{y}$ por tanto permiten mejorar la ganancia obtenida.

\section{Agradecimientos}

Este trabajo ha sido financiado por la Generalidad Valenciana, MINECO y Comisión Europea con los proyectos PROMETEO/2009/ 074, TEC2011-29120-C05-01 y NAVOLCHI 288869, respectivamente. 